\title{
Investigation and repair plan for abraded steel bridge piers: case study from Japan
}

\section{Pang-jo Chun PhD}

Project Associate Professor, Institute of Engineering Innovation, School of Engineering, University of Tokyo, Tokyo, Japan (corresponding author: chun@i-con.t.u-tokyo.ac.jp) (Orcid:0000-0002-9755-8435)

Keiji Tsukada PhD

Professor, Department of Electrical and Communication Engineering,

Okayama University, Okayama, Japan
Masahiro Kusumoto MSC

Director, Dai-ichi Consultants Co. Ltd, Kochi, Japan

Kazuaki Okubo PhD

Assistant Professor, Graduate School of International Cultural Studies, Tohoku University, Sendai, Japan

This paper reports on a forensic investigation following the collapse of the Iwama Ohashi Bridge over the Shimanto River in Japan. The central section of the bridge failed in 2017 when a steel pier buckled. The investigation included thickness measurements using a new ultrasensitive magnetic non-destructive device. This revealed that the buckled pier and other bridge piers had been significantly abraded by fast-moving gravel. As a result, the steel plate of the downstream faces near the riverbed was considerably thinner than the upstream faces. The measurements indicated that the other piers were also likely to buckle in the near future if left unattended. This paper describes the details of the investigation and discusses a potential repair and reinforcement plan.

$\begin{array}{ll}\text { Notation } & \\ \boldsymbol{B}_{\text {eddy }} & \text { magnetic field component } \\ \boldsymbol{B}_{\text {mag }} & \text { magnetisation term } \\ \boldsymbol{B}_{\mathrm{n}} & \text { net magnetic field vector } \\ I_{0} & \text { induction current } \\ k_{1} \text { and } k_{2} & \text { constants of proportionality } \\ L & \text { inductance of circuit } \\ R & \text { resistance of the circuit } \\ \omega & \text { angular frequency }\end{array}$

\section{Introduction}

Typhoons and heavy rains often cause rivers to swell considerably. Therefore, many bridges that cross such rivers are low-water bridges, which are designed to be used when the water flow is low and become submerged when the water level rises. To reduce the resistance against the flow of water when the water level is high, such bridges typically do not have parapets. Low-water bridges are favourable in that they can be built relatively quickly and inexpensively on lower ground and in shorter lengths compared to conventional bridges. Of the approximately 400 low-water bridges in Japan, 47 of them are across the Shimanto River. The Shimanto River flows through the Kochi Prefecture in Japan and is the longest river on the Shikoku Island $(196 \mathrm{~km})$, with a watershed of $2270 \mathrm{~km}^{2}$.

Here, the authors aim to investigate the collapse of one such lowwater bridge across the Shimanto River: the Iwama Ohashi Bridge. Figure 1 shows the bridge before the incident; it does not have parapets, as is the characteristic of low-water bridges as mentioned earlier. On 11 November 2017, the steel bridge pier near the centre of the Iwama Ohashi Bridge buckled and its central span collapsed, as shown in Figure 2 (Anon, 2018).

The buckled piers will be replaced. At the same time, other piers may also face the risk of similar buckling collapse and the authors want to prevent this. Therefore, the authors have measured the residual thickness by using a newly developed ultrasensitive magnetic non-destructive method, extremely low-frequency eddy current testing (Elect), and propose a repair and reinforcement plan in this research.

\section{Iwama Ohashi Bridge}

The Iwama Ohashi Bridge was built in 1963. It is $120 \mathrm{~m}$ long and $3 \mathrm{~m}$ wide. It is a simple prestressed-concrete-deck bridge with ten spans on steel-pile-bent piers. As mentioned in Section 1, it is a low-water bridge that becomes submerged under water when the water level rises in incidents such as flooding. The cross-sectional schematic diagram in Figure 3 shows that the bridge does not have parapets in order to minimise the water resistance when submerged. Figure 4 shows the side and top views of the bridge.

The Iwama Ohashi Bridge is one of the most important facilities, and it serves as a critical traffic point in the road network in the region. Many tourists visit the bridge every year, and it often appears on television commercials or advertisement posters. On 11 November 2017, the steel piers (P7) near the centre of the bridge buckled and the central span collapsed. Even though Iwama Ohashi Bridge was being inspected, similar damage cases were not reported in the past, so the inspection engineer overlooked the damage. Owing to its importance, the bridge must be repaired promptly. In addition to replacing the buckled pier, it must be decided whether countermeasures should be taken on the other piers. Herein, the authors evaluate the cause of the damage and present a potential repair strategy.

\section{Thickness measurements}

It is suspected that the buckling occurred because the steel piers had grown thin. It is necessary to measure the thicknesses of other steel piers to evaluate the risk of similar collapse by buckling. 


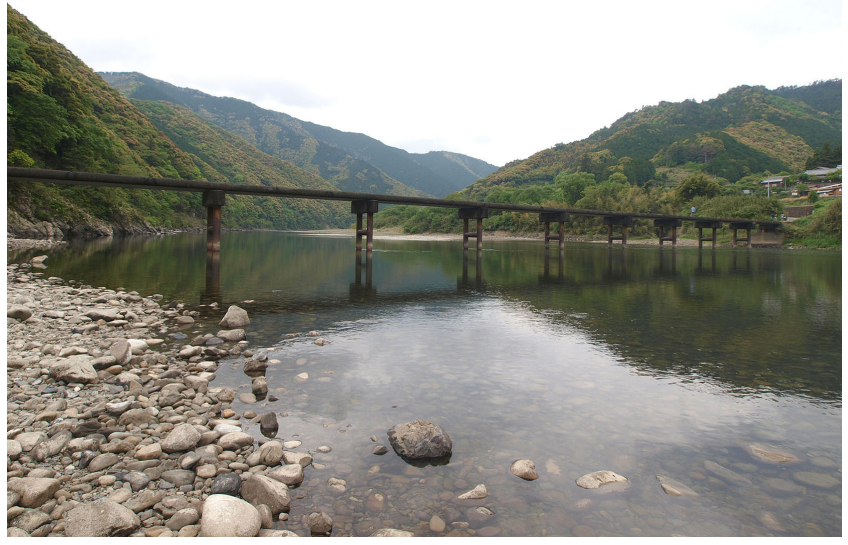

Figure 1. Photograph of the Iwama Ohashi low-water bridge that collapsed

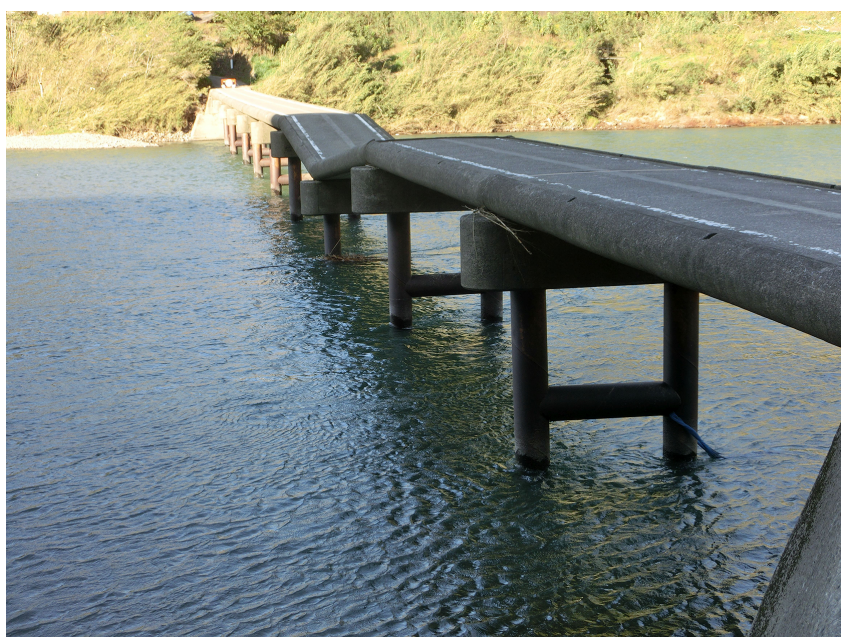

Figure 2. Collapse of the Iwama Ohashi Bridge at its centre

\subsection{Conventional techniques}

A micrometer (Figure 5) or an ultrasonic device (Figure 6) is generally used for such measurements. A micrometer measures the thickness of a specimen that is clamped between A and B, as shown in Figure 5. The device must be in physical contact with both sides of the target material. However, it is impossible to access the inside of the steel piers of the bridge to take the measurements. Therefore, this device cannot be used for this case.

The ultrasonic device can be used to measure the thickness of a target material based on the time required for an ultrasonic wave deployed from a probe to propagate through the material, bounce back from the opposite side of the specimen and return to the probe (Krautkrämer and Krautkrämer, 1990). To use this device, the surface of the target material must be smooth. However, the piers

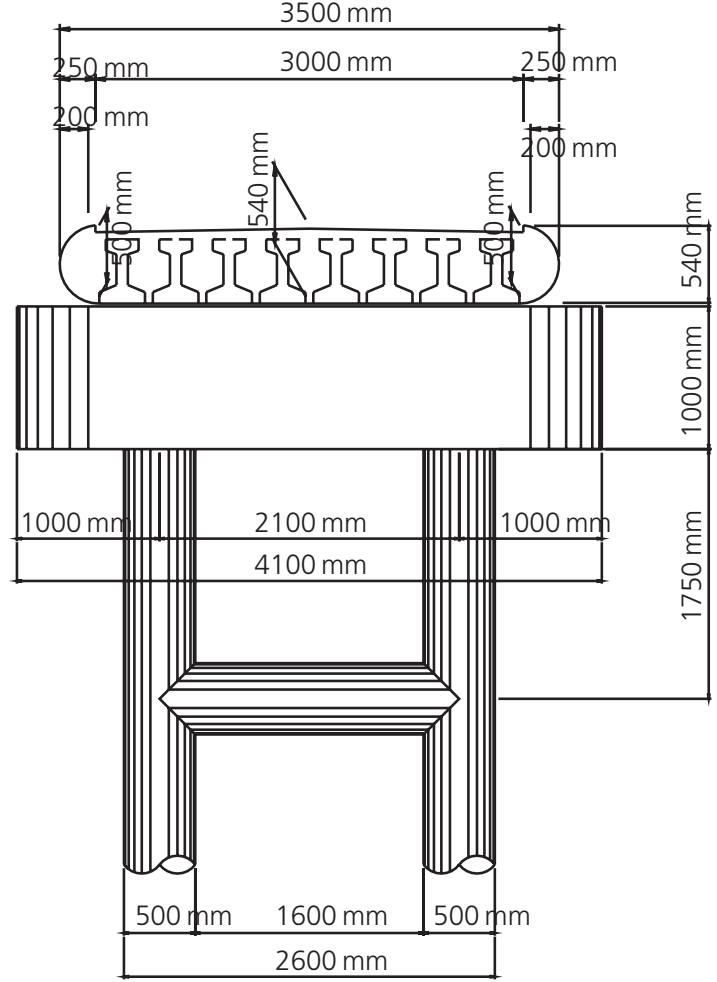

Figure 3. Cross-sectional view of the Iwama Ohashi Bridge

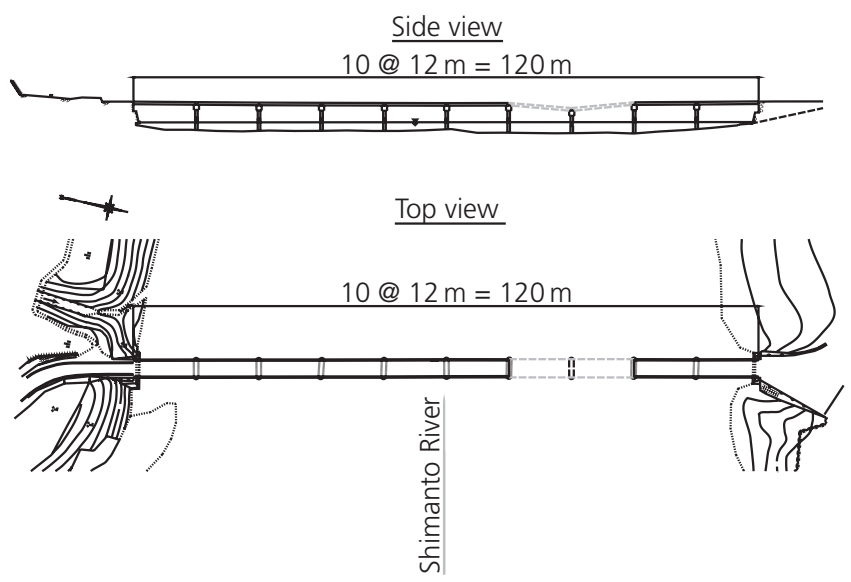

Figure 4. Side and top views of the Iwama Ohashi Bridge

of the Iwama Ohashi Bridge were corroded with rust. Therefore, surface preparation (i.e. scraping the submerged surface) would be necessary. Unfortunately, this process is highly time consuming, presents a risk of the corrosion products removed from the surface contaminating the river environment and can be done on only one side of the target material because it is impossible to access inside the steel piers. In addition, it is very difficult to do surface preparation for the underwater structure. Thus, ultrasonic measurements are not suitable for this application. 


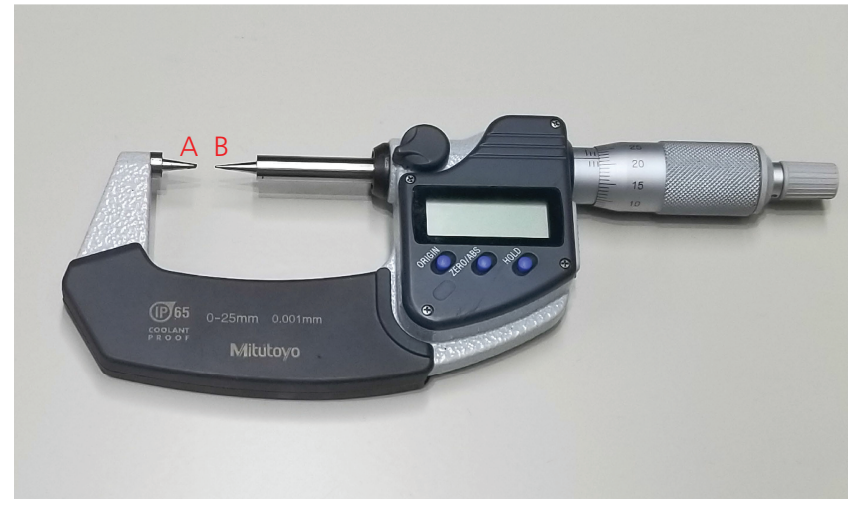

Figure 5. Photograph of a micrometer

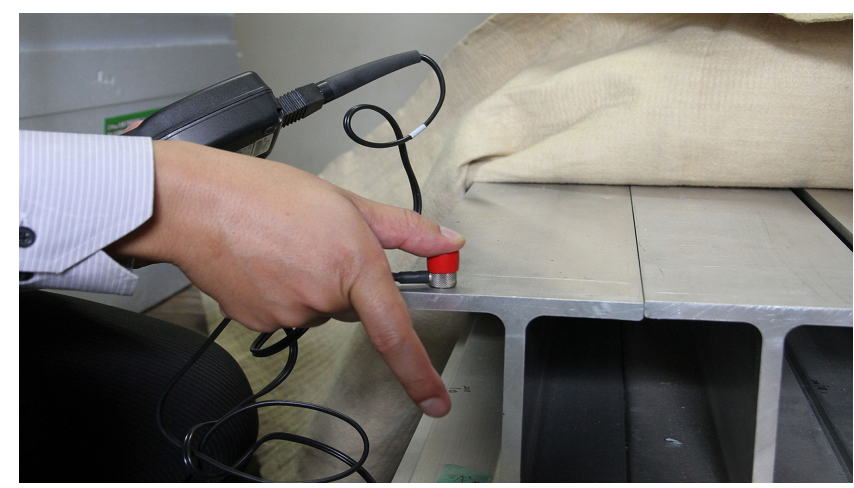

Figure 6. Plate thickness measurements performed using an ultrasonic device

\subsection{Extremely low-frequency eddy current testing}

As conventional means, such as using a micrometer or an ultrasonic device, are not suitable to measure accurately the steel thickness in this case, the authors propose a technique called Elect. Elect can be used to measure plate thickness on the basis of magnetism and can determine the thickness of the undamaged addresses the limitations of the conventional instruments: this approach does not require surface preparation or scraping on both addition, magnetism does not require a medium as an ultrasonic wave does; thus, non-contact measurements are possible.

The authors have previously utilised eddy current testing to detect various types of defects - for example, backside cracks in aluminium plates (Kiwa et al., 2007, 2009) and stainless-steel plates (Hayashi et al., 2008) and backside pits on steel plates (Tsukada et al., 2010). In addition, the authors have previously described a method for thickness measurement utilising eddy current testing (Tsukada et al., 2016) and developed the device shown in Figure 7, which enables field measurement based on theory. portion buried inside the corroded portion of a material. Elect sides of the material as the surface need not be smooth. In

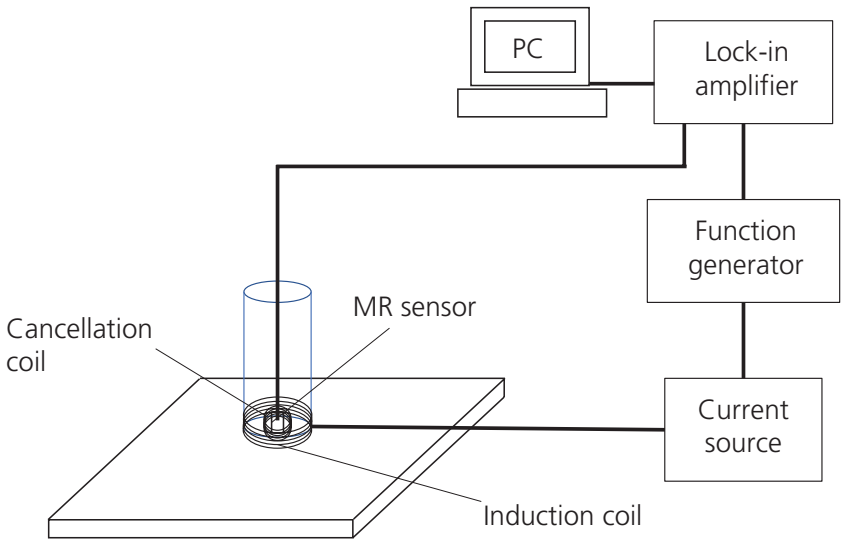

Figure 7. Schematic diagram of the magnetic measurement system. MR, magnetic resistance; PC, personal computer

The Elect probe is waterproof, and the measurement system was used to evaluate the steel piers underwater, as shown in Figure 8. The system consists of an induction coil that applies an alternating current (AC) magnetic field to an object, an anisotropic magnetic resistance sensor (Honeywell, HMC1001) to detect the normal magnetic component, a small cancellation coil near the sensor, a lock-in amplifier and a current source controlled by a function generator. The cancellation coil was used to minimise the magnetic field near the sensor to increase the signalto-noise ratio (SNR) because the applied magnetic field is stronger than the magnetic field generated by the eddy current of the object, particularly in the low-frequency range. The cancellation coil was wound around the magnetic resonance sensor with ten turns (optimised to maximise the SNR) and was $10 \mathrm{~mm}$ long and $1.5 \mathrm{~mm}$ wide. The cancellation coil was connected in series with the induction coil such that they were in phase. The circular induction coil had a $26 \mathrm{~mm}$ diameter and 50 turns; it was driven with an $\mathrm{AC}$ of $0 \cdot 1 \mathrm{~A}$ at frequencies ranging from $1 \mathrm{~Hz}$ to $1 \mathrm{kHz}$.

The theory developed by Tsukada et al. (2016) is outlined here for the convenience of the reader. The net magnetic field vector $\boldsymbol{B}_{\mathrm{n}}$ contains not only the magnetic field component $\boldsymbol{B}_{\text {eddy }}$ derived from the eddy current, but also a magnetisation term $\left(\boldsymbol{B}_{\mathrm{mag}}\right)$ based on the magnetisation characteristics because of the high permeability of iron steel. The eddy current of a conductive plate can be considered a series circuit with a resistance of $R$ and an inductance of $L$ that is driven by an electromotive force based on Faraday's law of electromagnetic induction. Therefore, the magnetic spectrum can be expressed as follows

$$
\begin{aligned}
\boldsymbol{B}_{\mathrm{n}} & =\boldsymbol{B}_{\text {eddy }}+\boldsymbol{B}_{\text {mag }} \\
& =-k_{1} I_{0} \frac{\omega^{2} L}{R^{2}+\omega^{2} L^{2}} \mathrm{e}^{i \omega t}-k_{2} I_{0} \frac{\omega^{2} R}{R^{2}+\omega^{2} L^{2}} \mathrm{e}^{i \omega t}+\boldsymbol{B}_{\text {mag }}
\end{aligned}
$$

1. 

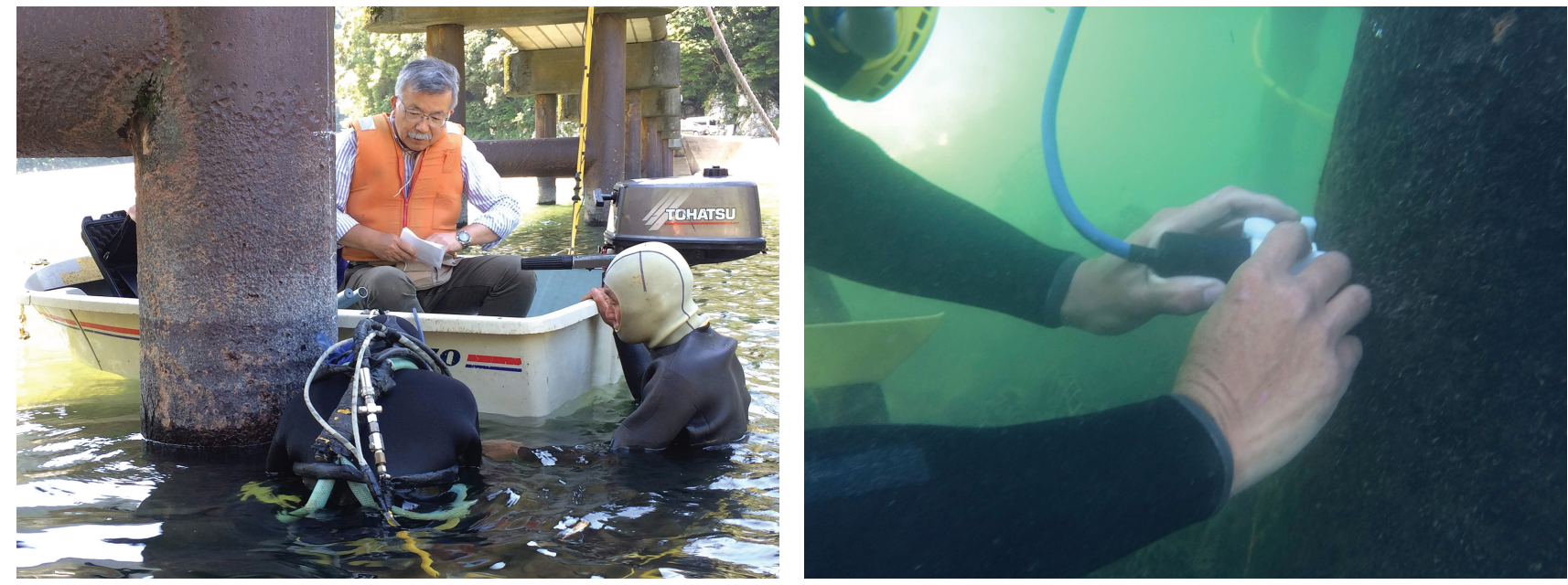

Figure 8. Measurement performed using the Elect system

where $I_{0}$ is the induction current, which is proportional to the magnetic induction field; $\omega$ is the angular frequency; and $k_{1}$ and $k_{2}$ are the constants of proportionality. When the measurement is performed with a constant $I_{0}$, the magnetic vector is shifted clockwise on the curve as the frequency is increased from the real axis. Therefore, the magnetic spectrum can be drawn as a trace of the net magnetic field vector $\boldsymbol{B}_{\mathrm{n}}$ at each frequency on a complex plane, as shown in Figure 9.

On the basis of the theory, the phase difference between the magnetic vectors developed by different frequencies, $f_{1}$ and $f_{2}$, is strongly correlated with the plate thickness. After testing various combinations of frequencies, $\left(f_{1}, f_{2}\right)=(3 \mathrm{~Hz}, 20 \mathrm{~Hz})$ and $\left(f_{1}, f_{2}\right)=$ $(50 \mathrm{~Hz}, 100 \mathrm{~Hz})$ are used in this study. Figure 10 shows the

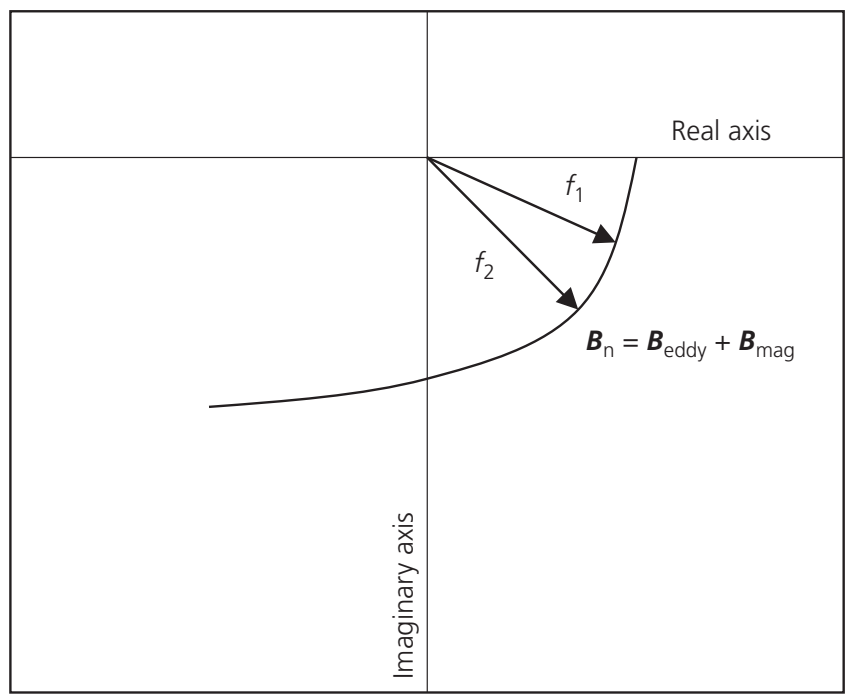

Figure 9. Basic concept of magnetic spectroscopy using magnetic vectors thickness dependence of the phase change of both frequency combinations obtained by a laboratory experiment beforehand. Based on the laboratory experiment, there is a clear relationship between the phase difference and a plate thickness less than $16 \mathrm{~mm}$. The lower frequencies of $\left(f_{1}, f_{2}\right)=(3 \mathrm{~Hz}, 20 \mathrm{~Hz})$ are appropriate for a thickness more than $3 \mathrm{~mm}$, and the higher frequencies of $\left(f_{1}, f_{2}\right)=(50 \mathrm{~Hz}, 100 \mathrm{~Hz})$ are appropriate for a thickness less than $3 \mathrm{~mm}$. The initial plate thickness of the steel piers of the Iwama Ohashi Bridge was $10 \mathrm{~mm}$, which is within the acceptable range. For a specific measurement procedure, first measure with $\left(f_{1}, f_{2}\right)=(3 \mathrm{~Hz}, 20 \mathrm{~Hz})$ to obtain the phase. If the phase is $1.5 \mathrm{rad}$ or larger, which means that the thickness is $3 \mathrm{~mm}$ or less, change to $\left(f_{1}, f_{2}\right)=(50 \mathrm{~Hz}, 100 \mathrm{~Hz})$ and measure again. By referring to this relationship, the plate thickness of a real structure can be determined based on the phase difference

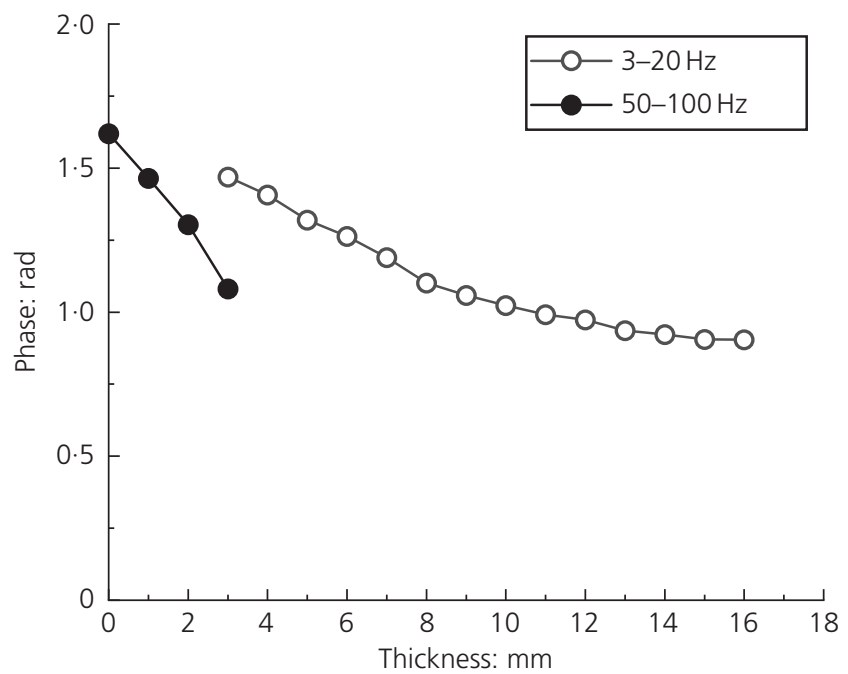

Figure 10. Thickness dependence of the phase change 
obtained accurately in field measurements. Moreover, the measurement time is short (about $10 \mathrm{~s}$ per point), which enables thorough measurements of the target material.
Note that steel materials have parameters of magnetic permeability and electric conductivity, which affect the thickness measurement results. However, this research uses the phase of the

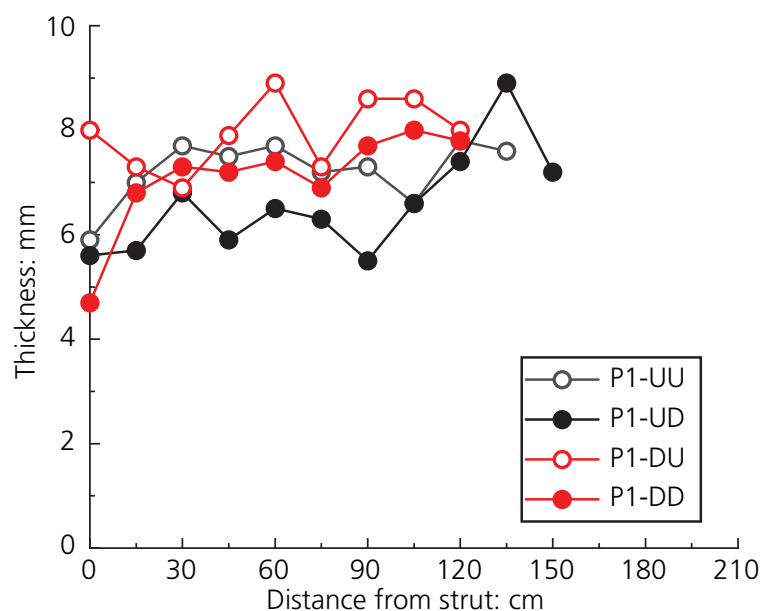

(a)

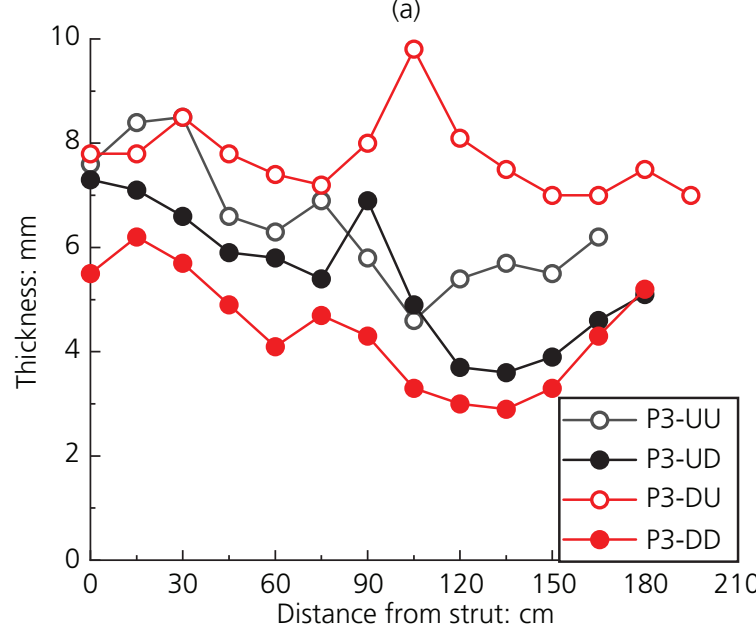

(c)

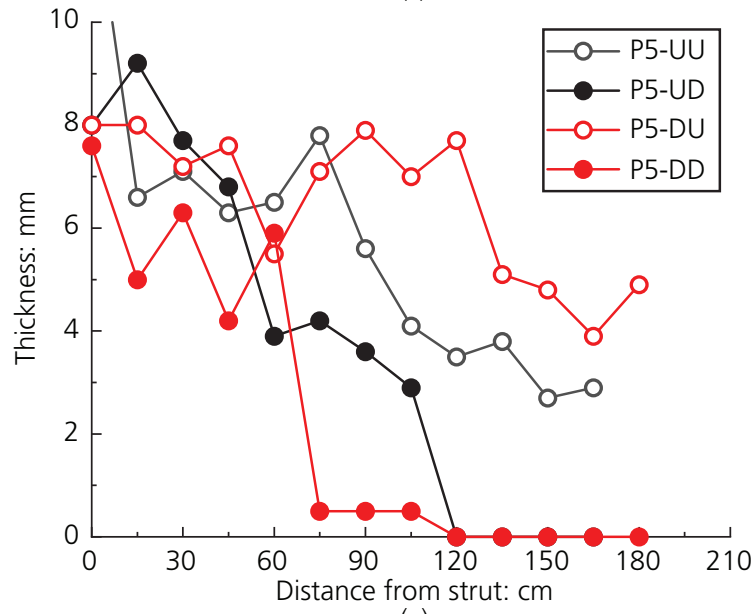

(e)

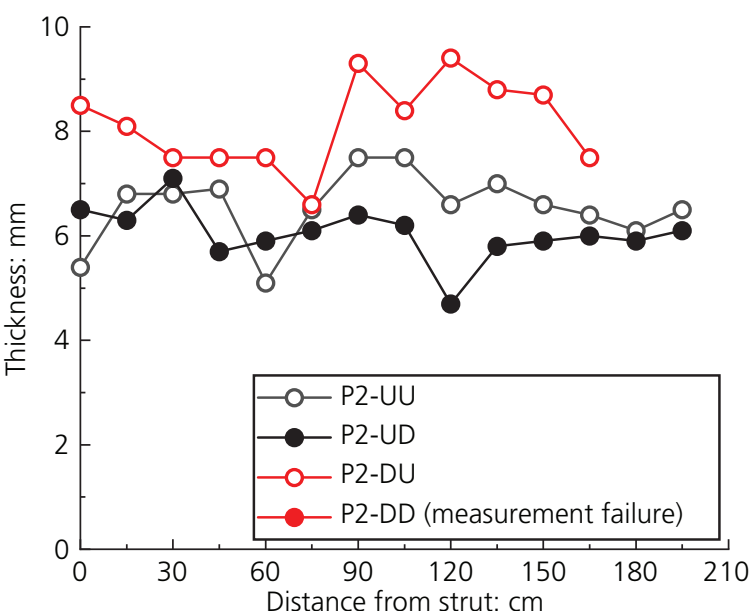

(b)

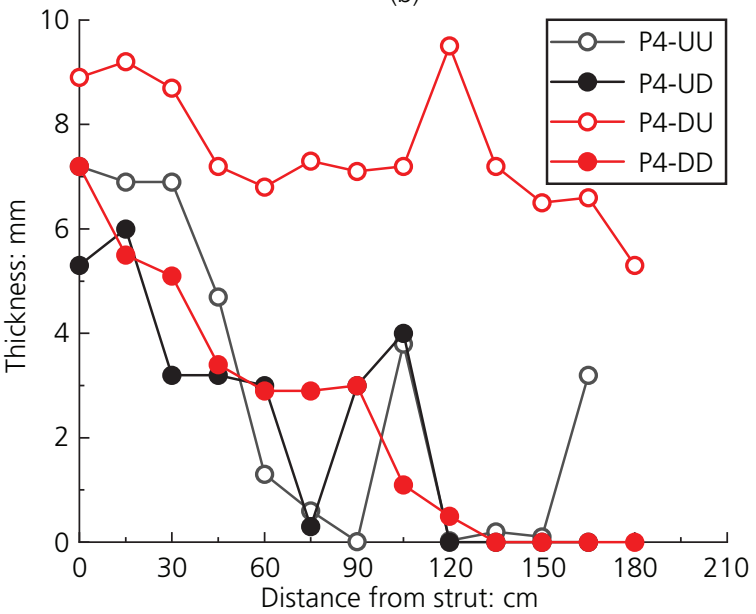

(d)

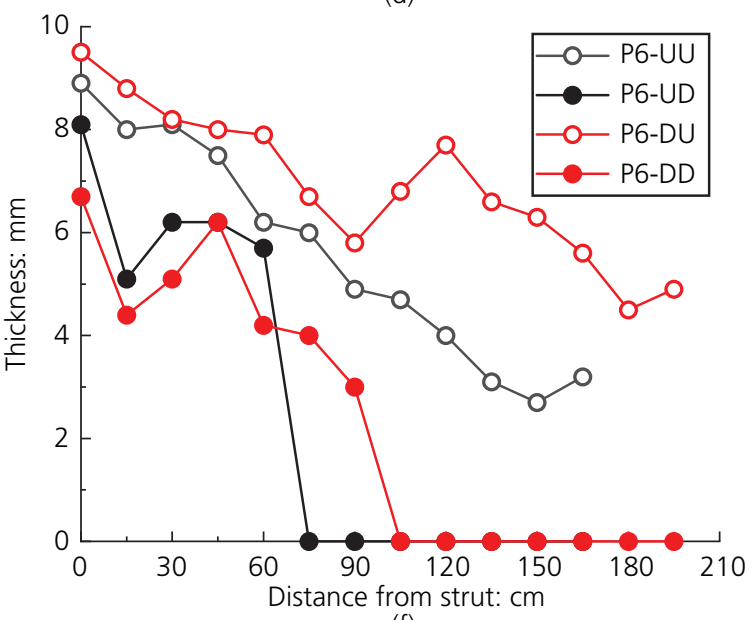

(f)

Figure 11. Results of thickness measurement of P1-P6 using Elect (the result for P2-DD is not shown because of a mechanical failure during the measurement). Thickness measurement results of: (a) P1, (b) P2, (c) P3, (d) P4, (e) P5 and (f) P6. A full-colour version of this figure can be found on the ICE Virtual Library (www.icevirtuallibrary.com) 
magnetic field vector difference between the two frequencies to cancel the influence of the magnetic permeability. As for electric conductivity, the influence was cancelled by performing calibration using a database of the relationship between material and phase at a position where the plate thickness can be confirmed by ultrasonic measurement.

\section{Measurement results}

The results of the Elect measurement conducted on 1 June 2018 are shown in Figure 11. The piers on the north side of the buckled pier, P7 (i.e. from P1 to P6), were examined; P8 and P9 were excluded because reinforcement work had already been undertaken on them.

Both the upstream and downstream faces on the upstream-side and downstream-side piers of P1-P6 were tested. In the marker labels in Figure 11, U represents the upstream side and D represents the downstream side. Moreover, the first letter indicates the side of the river on which the particular pier is located, whereas the second letter indicates the side of the pier's surface. For example, P3-UD indicates the downstream face of the upstream-side pier of P3. This naming convention is shown in Figure 12.

Measurements were taken at several points on each pier starting from immediately below the strut down to the bottom of the river in intervals of $15 \mathrm{~cm}$ (Figure 13). The water surface was $45-60 \mathrm{~cm}$ below the struts.

For piers $\mathrm{P} 1$ to $\mathrm{P} 3$, measurements were also collected in the circumferential direction; the results are shown in Figure 14. The plate thickness on pier P3 was significantly reduced on the downstream side compared to the upstream side. However, the thickness decrease was more consistent around the entire circumference on piers $\mathrm{P} 1$ and $\mathrm{P} 2$.

The results shown in Figures 11 and 14 indicate that the steel thickness decreased and became significantly worse from piers P1 to P6. The thickness decrease was most significant near the bottom of the river, particularly on the downstream side. Figure 15 shows the buckled pier, which was brought back to the laboratory for further investigation. On the surface of the buckled pier near the river bottom, there were many pockmarks that were similar in size to the gravel on the riverbed. Thus, it was hypothesised that the gravel accelerated by the current contributed to the measured reduction in steel thickness. Pier P6 was more damaged than pier P1, implying that the damage was more severe near the centre of the river, where the current was stronger than

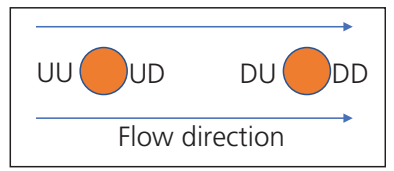

Figure 12. Naming convention for the piers

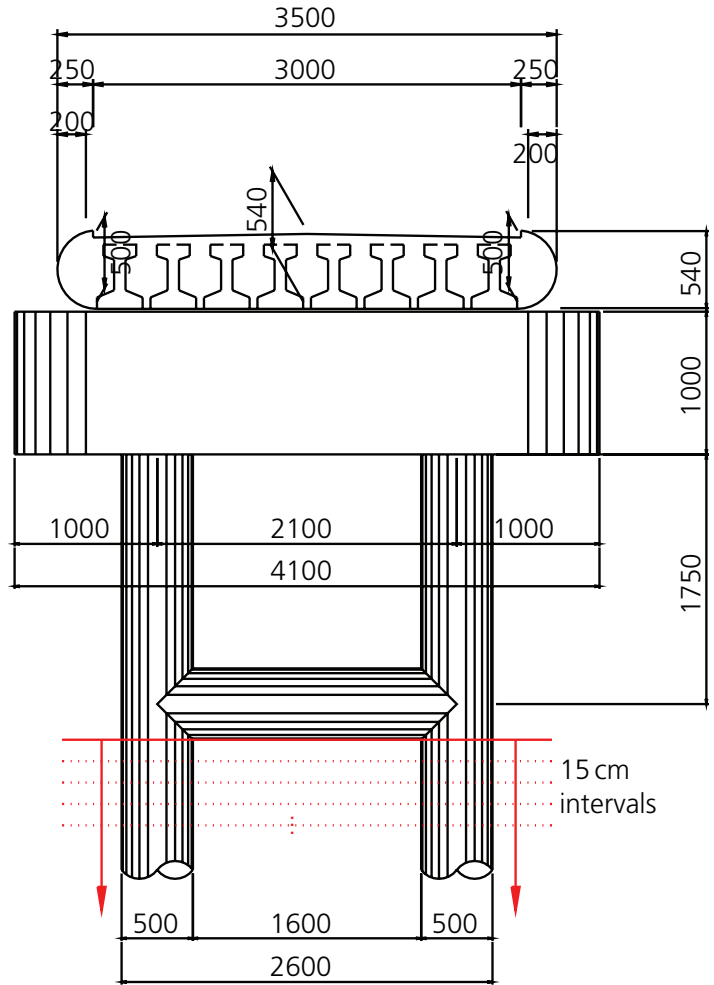

Figure 13. Measuring points set in intervals of $15 \mathrm{~cm}$ from the strut (other units in $\mathrm{mm}$ )

near the riverside. These observations suggest that the observed pockmarks were caused by a Karman vortex around the piers, as shown in Figure 16. Owing to such a water flow pattern, the gravel deposited on the downstream side of the pier would be stirred up by the Karman vortex and hit the downstream side of the pier surface. This effect would damage the passivation film on the steel and accelerate the corrosion of the piers.

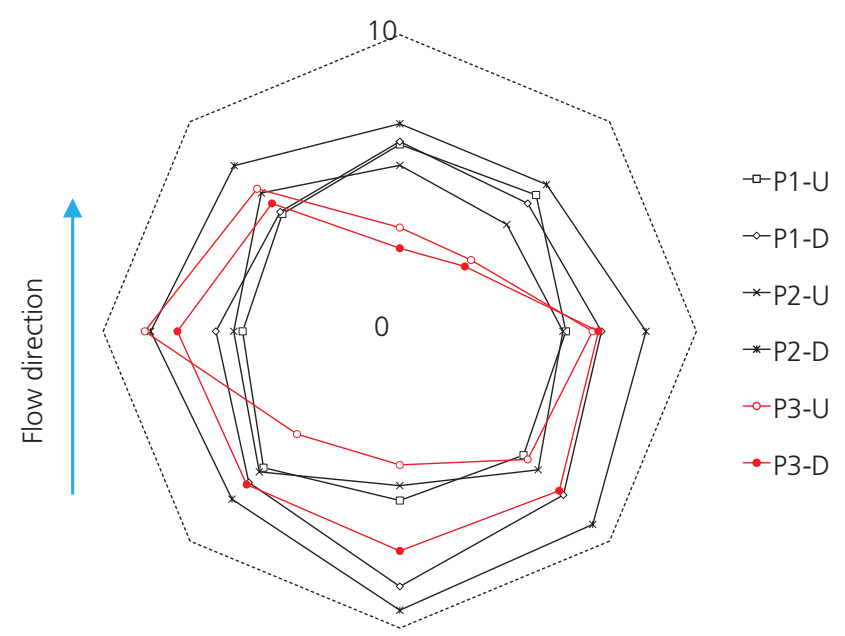

Figure 14. Thickness measurement results in the circumferential direction 


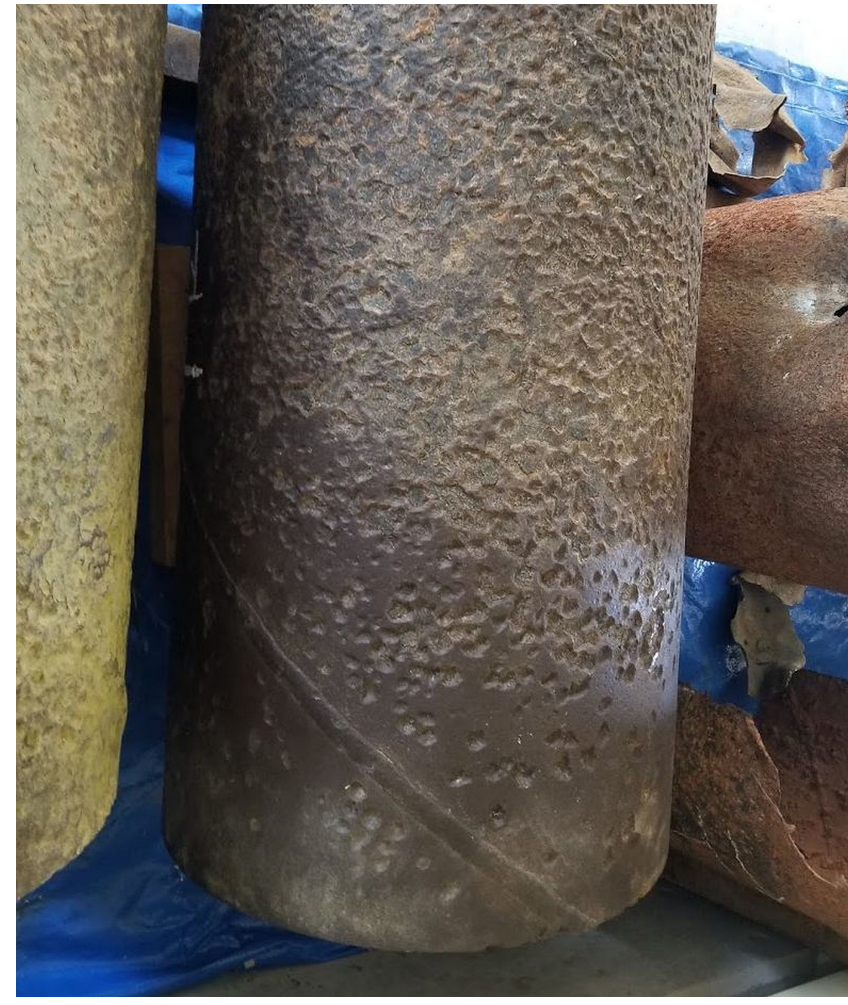

Figure 15. Buckled pier above the buckled portion. Many pockmarks are evident

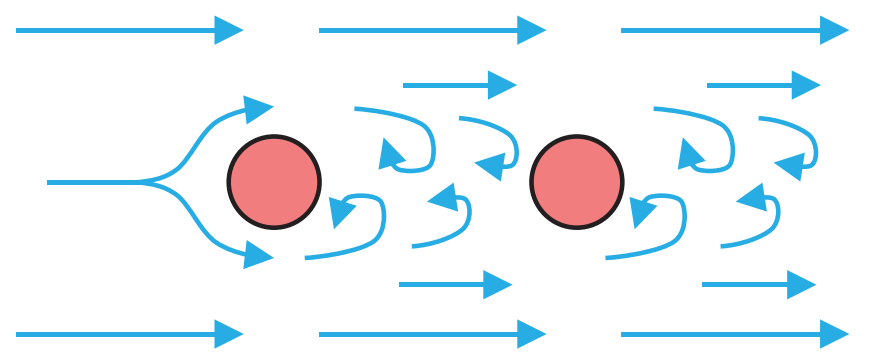

Figure 16. Current flow diagram around the piers

Furthermore, as for the upstream face, the downstream-side piers were less damaged than the upstream-side piers. This was probably because the downstream-side piers were located in the turbulent flow caused by the upstream-side piers. Piers P4-P6 were severely damaged on the downstream face near the river bottom, and the presence of similar pitting was visually confirmed on some of them. Thus, it was concluded that these particular piers were at high risk of collapse as the load of the bridge was supported only by the steel on the upstream face.

\section{Recommended repair plan}

On the basis of the findings, the authors propose a plan to repair the damage and counter future incidents. In addition to replacing the buckled pier, P7, the other severely damaged piers P4-P6

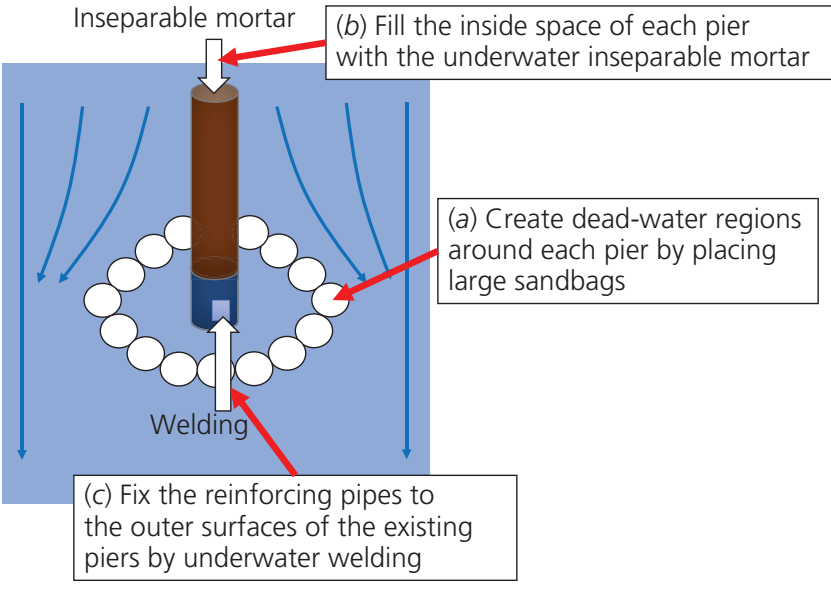

Figure 17. Schematic diagram of proposed repair work

require reinforcement work. Regarding P1-P3, it was diagnosed that reinforcement work is unnecessary, as plate thickness remains more than half even in the thinnest section.

The superstrengthening pile-bent (SSP) method is one of the most commonly used methods for reinforcing steel piers in Japan (OSC, 2018). The SSP method involves pressing the reinforcing steel pier onto the outer side of the existing pier and filling the gap between the reinforcing pier and existing pier with mortar to reinforce it. However, the SSP approach is not suitable for the Iwama Ohashi Bridge because the bridge clearance height is too small to fit the press-fitting machine, the struts placed between the piers prevent the proper application of the reinforcing piers and the riverbed (comprising gravel and cobblestones) is too unstable to press reinforcements.

Therefore, the authors propose an alternative reinforcement plan as in Figure 17: first, create dead-water regions around each pier by placing large sandbags. Next, fill the inside space of each pier with the underwater inseparable mortar to prevent them from buckling. Finally, fix the reinforcing pipes to the outer surfaces of the existing piers by underwater welding. Through this approach, the strength of the pier can be restored without spoiling the surrounding scenery and similar buckling incidents can be prevented in the future.

\section{Conclusion}

In this paper, the authors discuss the Iwama Ohashi Bridge, which is currently unusable because of a buckling incident. The authors present measurements of the plate thicknesses on the piles obtained using the Elect system that they developed. On the basis of the findings, the authors propose a suitable counterplan and methods to reinforce the damaged piers. This plan involved using underwater inseparable mortar and applying reinforcing pipes on the outer surfaces of the existing piers through underwater welding. The proposed countermeasures are expected to restore strength to the damaged bridge piers and prevent future buckling incidents. This technique can also be applied to various types of 
steel structures other than steel-pile-bent piers, such as pillars that support landing stages.

As the next step in research, the authors intend to study the connection between the Karman vortex and the size of the gravel and investigate the speed and energy at which the current moves the gravel to study the damage mechanism of piers from a hydrodynamic viewpoint.

\section{Acknowledgement}

This work was supported by the Council for Science, Technology and Innovation, 'Crossministerial Strategic Innovation Promotion Program (SIP), Infrastructure Maintenance, Renovation, and Management' (funding agency: Japan Science and Technology Agency).

\section{REFERENCES}

Anon (2018) Iwama Ohashi bridge in Shimanto City, Kochi Prefecture is totally closed for traffic. Kochi Shimbun, 12 November. See https:// www.kochinews.co.jp/article/138653/ (accessed 12/11/2018).
Hayashi T, Yamada H, Kiwa T, Tsukada K and Tamazumi M (2008) Detection of the weak magnetic properties change of stainless-steel welding parts by low frequency magnetic imaging. Journal of Applied Physics 103(7): 07E923, https://doi.org/10.1063/1.2837628.

Kiwa T, Kawata T, Yamada H and Tsukada K (2007) Fourier-transformed eddy current technique to visualize cross-sections of conductive materials. NDT\&E International 40(5): 363-367, https://doi.org/ 10.1016/j.ndteint.2007.01.006.

Kiwa T, Hayashi T, Kawasaki Y, Yamada H and Tsukada K (2009) Magnetic thickness gauge using a Fourier transformed eddy current technique. NDT\&E International 42(7): 606-609, https://doi.org/ 10.1016/j.ndteint.2009.04.003.

Krautkrämer J and Krautkrämer H (1990) Ultrasonic Testing of Materials. Springer, New York, NY, USA.

OSC (Oriental Shiraishi Corporation) (2018) Pier-rifre Method. OSC, Tokyo, Japan. See http://www.orsc.co.jp/english/tec/con05_9.html (accessed 11/11/2018).

Tsukada K, Yoshioka M, Kawasaki Y and Kiwa T (2010) Detection of back-side pit on a ferrous plate by magnetic flux leakage method with analyzing magnetic field vector. NDT\&E International 43(4): 323-328, https://doi.org/10.1016/j.ndteint.2010.01.004.

Tsukada K, Haga Y, Morita K et al. (2016) Detection of inner corrosion of steel construction using magnetic resistance sensor and magnetic spectroscopy analysis. IEEE Transactions of Magnetics 52(7): article 6201504, https://doi.org/10.1109/TMAG.2016.2530851.

\section{How can you contribute?}

To discuss this paper, please email up to 500 words to the editor at journals@ice.org.uk. Your contribution will be forwarded to the author(s) for a reply and, if considered appropriate by the editorial board, it will be published as discussion in a future issue of the journal.

Proceedings journals rely entirely on contributions from the civil engineering profession (and allied disciplines). Information about how to submit your paper online is available at www.icevirtuallibrary.com/page/authors, where you will also find detailed author guidelines. 